\title{
ON THE ASYMPTOTIC BEHAVIOUR OF THE SOLUTIONS TO A CLASS OF SECOND ORDER NONLINEAR DIFFERENTIAL EQUATIONS
}

\author{
OLIVIA LIPOVAN \\ Department of Mathematics, West University, Bd. V. Parvan 4, Timisoara 1900, Romania
}

(Received 7 March, 2002; accepted 10 April, 2002)

\begin{abstract}
For a class of second order nonlinear differential equations, sufficient conditions are presented to ensure that some, respectively all solutions are asymptotic to lines.
\end{abstract}

2000 Mathematics Subject Classification. 34E10.

1. Introduction. The asymptotic properties of the solutions to the second order nonlinear differential equation

$$
u^{\prime \prime}=f(t, u), \quad t \geq 1,
$$

where $f \in C([1, \infty) \times \mathbb{R}, \mathbb{R})$ have been widely investigated by Cohen [1], Constantin [2], Dannan [5], Fan Wei Meng [6], S. Rogovchenko and Y. Rogovchenko [12], Tong [13] and Trench [14]. Using integral inequalities, these papers were concerned with sufficient conditions which ensure that all solutions of (1) will approach those of $u^{\prime \prime}=0$. More precisely, sufficient conditions were given such that the solutions of (1) satisfy

$$
u(t)=a t+o(t) \quad \text { as } \quad t \rightarrow \infty
$$

i.e.

$$
\frac{u(t)}{t} \rightarrow a \text { as } t \rightarrow \infty, \quad \text { for some } a \in \mathbb{R} .
$$

In this paper we also investigate conditions ensuring that solutions of (1) behave asymptotically like linear functions. In Theorem 1 we give sufficient conditions guaranteeing that for all $a, b \in \mathbb{R}$, there is a solution to (1) with

$$
u(t)=a t+b+o(1) \quad \text { as } \quad t \rightarrow \infty .
$$

Theorem 2 provides a setup in which all solutions to (1) have this property. In our approach a key role is played by the fixed point technique and we will also rely on some results from the papers cited above. Examples are provided to illustrate the applicability of our results, e.g. to sublinear Emden-Fowler equations.

\section{Main results.}

THEOREM 1. Consider equation (1) with $f \in C([1, \infty) \times \mathbb{R}, \mathbb{R})$ satisfying

$$
|f(t, u)| \leq h_{1}(t) \cdot g\left(\frac{|u|}{t}\right)+h_{2}(t), \quad t \geq 1, u \in \mathbb{R}
$$


where $g \in C\left(\mathbb{R}_{+}, \mathbb{R}_{+}\right), h_{1}, h_{2} \in C\left([1, \infty), \mathbb{R}_{+}\right)$and

$$
\int_{1}^{\infty} s h_{i}(s) d s<\infty, \quad i=\overline{1,2}
$$

Then, for every $a, b \in \mathbb{R}$ equation (1) has a solution $u(t)$ defined on an interval $[T, \infty)$ $(T=T(a, b))$, such that $u(t)$ is asymptotic to the line at $+b$ for $t \rightarrow \infty$, i.e.

$$
u(t)-(a t+b) \rightarrow 0 \text { for } t \rightarrow \infty .
$$

Proof. Let us first observe that

$$
\int_{1}^{\infty} s h_{i}(s) d s<\infty \quad \text { forces } \int_{1}^{\infty} \int_{t}^{\infty} h_{i}(s) d s d t<\infty \quad \text { for } \quad i=\overline{1,2} .
$$

Take $a \in \mathbb{R}$ and denote

$$
y(t)=u(t)-a t, \quad t \geq 1 .
$$

Then (1) becomes

$$
y^{\prime \prime}(t)=f(t, y(t)+a t) .
$$

We notice that if for every $b \in \mathbb{R}$, equation (5) has a solution $y(t)$ such that

$$
y(t) \rightarrow b \quad \text { as } \quad t \rightarrow \infty
$$

our theorem is proved.

Let

$$
M=\sup _{0 \leq \tau \leq|a|+|b|+1}|g(\tau)|
$$

and choose $T \geq 1$ sufficiently large such that

$$
\int_{T}^{\infty}\left[\int_{s}^{\infty} h_{1}(r) d r\right] d s<\frac{1}{2 M} \text { and } \int_{T}^{\infty}\left[\int_{s}^{\infty} h_{2}(r) d r\right] d s<\frac{1}{2}
$$

Consider now the Banach space

$$
X=\{x \in C([T, \infty), \mathbb{R}): x(t) \text { is bounded }\}
$$

with the supremum norm.

Let $K \subset X$ be the closed, convex and bounded set

$$
K=\{x \in X:|x(t)-b| \leq 1 \quad \text { for } t \geq T\},
$$

and define the map $F: K \rightarrow X$,

$$
(F x)(t)=b+\int_{t}^{\infty}\left(\int_{s}^{\infty} f(r, x(r)+a r) d r\right) d s, \quad t \geq T .
$$


As a consequence of hypothesis (2) and the relations (4) we infer the continuity of $F: K \rightarrow X$ from the Lebesgue dominated convergence theorem.

Let us show that $F$ is compact and $F(K) \subset K$. Taking $x \in K$, we have that

$$
\begin{aligned}
|(F x)(t)-b| & \leq \int_{t}^{\infty}\left(\int_{s}^{\infty}|f(r, x(r)+a r)| d r\right) d s \\
& \leq \int_{t}^{\infty} \int_{s}^{\infty}\left[h_{1}(r) g\left(\left|\frac{x(r)}{r}+a\right|\right)+h_{2}(r)\right] d r d s \\
& \leq M \cdot \int_{t}^{\infty}\left(\int_{s}^{\infty} h_{1}(r) d r\right) d s+\int_{t}^{\infty}\left(\int_{s}^{\infty} h_{2}(r) d r\right) d s \\
& \leq \frac{1}{2}+\frac{1}{2}=1, \quad t \geq T,
\end{aligned}
$$

and the last relation shows that $F(K) \subset K$.

In order to show that $F$ is compact, let $\left\{x_{n}\right\}_{n \geq 1}$ be a sequence in $K$ and denote

$$
f_{n}(s)=\int_{s}^{\infty} f\left(r, x_{n}(r)+a r\right) d r, \quad n \geq 1, s \geq T .
$$

Then the sequence $\left\{f_{n}\right\}_{n \geq 1}$ is bounded in $L^{1}([T, \infty), \mathbb{R})$ and

$$
\lim _{p \rightarrow \infty} \int_{p}^{\infty}\left|f_{n}(s)\right| d s=0
$$

uniformly in $n \geq 1$.

Indeed,

$$
\begin{aligned}
\int_{p}^{\infty}\left|f_{n}(s)\right| d s & \leq \int_{p}^{\infty}\left(\int_{s}^{\infty}\left|f\left(r, x_{n}(r)+a r\right)\right| d r\right) d s \\
& \leq \int_{p}^{\infty} \int_{s}^{\infty}\left[h_{1}(r) \cdot g\left(\left|\frac{x_{n}(r)}{r}+a\right|\right)+h_{2}(r)\right] d r d s \\
& \leq M \cdot \int_{p}^{\infty}\left(\int_{s}^{\infty} h_{1}(r) d r\right) d s+\int_{p}^{\infty}\left(\int_{s}^{\infty} h_{2}(r) d r\right) d s
\end{aligned}
$$

so that the boundedness of $\left\{f_{n}\right\}_{n \geq 1}$ and relation (6) are ensured by (4).

We want to show that

$$
\left\|\tau_{\delta}\left(f_{n}\right)-f_{n}\right\|_{L^{1}([T, \infty), \mathbb{R})} \stackrel{\delta \rightarrow 0}{\longrightarrow} 0
$$

uniformly in $n \geq 1$, where $\tau_{\delta}$ is the translation by $\delta$ to the left:

$$
\left(\tau_{\delta} f\right)(t)=f(t+\delta), \quad t \geq 1, \delta>0 .
$$


We have the following estimate

$$
\begin{aligned}
\int_{T}^{\infty}\left|f_{n}(s+\delta)-f_{n}(s)\right| d s & \leq \int_{T}^{\infty}\left(\int_{s}^{s+\delta}\left|f\left(r, x_{n}(r)+a r\right)\right| d r\right) d s \\
& \leq \int_{T}^{\infty} \int_{s}^{s+\delta}\left[h_{1}(r) \cdot g\left(\left|\frac{x_{n}(r)}{r}+a\right|\right)+h_{2}(r)\right] d r d s \\
& \leq M \cdot \int_{T}^{\infty}\left(\int_{s}^{s+\delta} h_{1}(r) d r\right) d s+\int_{T}^{\infty}\left(\int_{s}^{s+\delta} h_{2}(r) d r\right) d s
\end{aligned}
$$

The above expression does not depend on $n \geq 1$ and, as

$$
\int_{s}^{s+\delta} h_{i}(r) d r \leq \int_{s}^{\infty} h_{i}(r) d r \quad \text { for } s \geq T \text { and } i=\overline{1,2},
$$

by (4) and the Lebesgue dominated convergence theorem we deduce that (7) is verified. Using (6)-(7) and the boundedness of $\left\{f_{n}\right\}_{n \geq 1}$ in $L^{1}([T, \infty), \mathbb{R})$, we apply Riesz's theorem (see [10]) to conclude that $\left\{f_{n}\right\}_{n \geq 1}$ is relatively compact in $L^{1}([T, \infty), \mathbb{R})$.

In view of the relation

$$
\left(F x_{n}\right)(t)=b+\int_{t}^{\infty} f_{n}(s) d s, \quad t \geq T, n \geq 1,
$$

we can easily see that $\left\{F x_{n}\right\}_{n \geq 1}$ is relatively compact in $K$. This proves that $F$ is a compact map.

We can apply Schauder's fixed point theorem $[\mathbf{8}]$ to deduce that $F$ has a fixed point $y \in K$. Therefore

$$
y(t)=b+\int_{t}^{\infty}\left(\int_{s}^{\infty} f(r, y(r)+a r) d r\right) d s, \quad t \geq T,
$$

which shows that $y(t)$ is a solution to (5) and

$$
\lim _{t \rightarrow \infty} y(t)=b .
$$

The proof is completed.

The following example illustrates the dependence of the initial point $T(a, b)$ on the constants $a, b$ in Theorem 1 .

EXAMPLE 1. Consider the equation (1) with

$$
f(t, x)=\left\{\begin{array}{l}
0, \quad t \geq 3, \\
2(3-t)\left(x+t^{2}\right)\left[\left(x+t^{2}\right)^{2}+\left(x+t^{2}\right)+1\right], \quad 1 \leq t<3, x \geq-t^{2}, \\
0, \quad 1 \leq t<3, x<-t^{2},
\end{array}\right.
$$


satisfying the hypotheses of Theorem 1 . Note that $x(t)=-10, t \geq 1$ is a solution to the equation, thus for $a=0, b=-10$, we have $T(0,-10)=1$.

On the other hand, taking $a=-7, b=13$, we get that

$$
x^{*}(t)= \begin{cases}\frac{1}{t-2}-t^{2}, & t \in(2,3], \\ -7 t+13, & t \in(3, \infty),\end{cases}
$$

is a solution to (1) for which $T(a, b)>2$. Indeed, since $f$ is locally Lipschitz in the spatial variable throughout the region

$$
\left\{(t, x): t \geq 3 \quad \text { or } \quad x \geq-t^{2} \quad \text { if } t \in[1,3]\right\},
$$

and all solutions are affine for $t \geq 3$, it is clear that $x^{*}$ is the only solution asymptotic to the line $13-7 t$ as $t \rightarrow \infty$.

The statement of Theorem 1 raises another question: are lines the only possible asymptotes for the solutions? The trivial example $u^{\prime \prime}=0$ shows that this is possible, though it is not always the case (see the example below).

EXAMPLE 2. Let us consider the nonlinear differential equation

$$
u^{\prime \prime}=\frac{3}{t^{5}} u^{2}, \quad t \geq 1
$$

By Theorem 1 we have that for each pair $(a, b) \in \mathbb{R}^{2}$ we can find a solution of the above equation which is asymptotic to $a t+b$ as $t \rightarrow \infty$. On the other hand, we observe that $u(t)=2 t^{3}$ is a solution of the equation which is not asymptotic to a line as $t \rightarrow \infty$.

Now we will analyse under which additional conditions all solutions of (1) are asymptotic to lines. For this to hold, it is first of all necessary that all solutions of (1) are global. By standard extension theorems (see [4] or [8]), all solutions of (1) are global if there exists $M_{0}>0$ such that $g(r)>0$ for $r \geq M_{0}$, and $g$ is nondecreasing on $\left[M_{0}, \infty\right)$ with

$$
\int_{M_{0}}^{\infty} \frac{d s}{g(s)}=\infty
$$

It turns out that adding the condition (8) for a nondecreasing function $g$ : $\left[M_{0}, \infty\right) \rightarrow(0, \infty)$ to the hypoteses of Theorem 1 , not only all solutions of (1) are globally defined, but they are all asymptotic to lines. To see this we will rely partly on previous investigations towards the asymptotic behaviour of the solutions for second order nonlinear differential equations.

TheOREM A. [12]. Suppose that $f \in C([1, \infty) \times \mathbb{R}, \mathbb{R})$ and there exist continuous functions $h_{1}, h_{2}:[1, \infty) \rightarrow \mathbb{R}_{+}, g: \mathbb{R}_{+} \rightarrow \mathbb{R}_{+}$, such that

$$
|f(t, u)| \leq h_{1}(t) \cdot g\left(\frac{|u|}{t}\right)+h_{2}(t), \quad t \geq 1, u \in \mathbb{R}
$$


where for $s>0$ the function $g(s)$ is nondecreasing,

$$
\int_{1}^{\infty} h_{i}(s) d s<+\infty, \quad i=1,2
$$

and if we denote

$$
G(x)=\int_{1}^{x} \frac{d s}{g(s)}
$$

then $G(+\infty)=+\infty$.

Then, any solution of equation (1) is of the form

$$
u(t)=a t+o(t) \quad \text { for } \quad t \rightarrow \infty
$$

where $a \in \mathbb{R}$.

Theorem A generalizes results obtained in [1], [2], [5], [6], [13], [14], and, though it does not ensure that all solutions are asymptotic to lines (see Example 3), it represents a starting point for our approach.

EXAMPLE 3. The equation

$$
u^{\prime \prime}=-\frac{1}{t^{2}}, \quad t \geq 1
$$

satisfies the hypotheses of Theorem A and has the general solution $u(t)=\ln t+a t+b$, $t \geq 1,(a, b \in \mathbb{R})$. All solutions are of the form $a t+o(t)$ for $t \rightarrow \infty$, but none is asymptotic to a line.

Let us now prove the following result.

THEOREM 2. Consider equation (1), where the function $f \in C([1, \infty) \times \mathbb{R}, \mathbb{R})$ satisfies the hypotheses of Theorem 1 , with the additional assumption: $g: \mathbb{R}_{+} \rightarrow(0, \infty)$ is nondecreasing with $\int_{1}^{\infty} \frac{d s}{g(s)}=\infty$.

Then, every solution $u(t)$ of (1) is asymptotic to a line for $t \rightarrow \infty$, i.e. $u(t)=a t+$ $b+o(1)$ for $t \rightarrow \infty$.

Proof. Let $x(t), t \geq 1$ be a fixed solution to (1). Then

$$
x(t)=x(1)+x^{\prime}(1)(t-1)+\int_{1}^{t}\left[\int_{1}^{s} f(r, x(r)) d r\right] d s, \quad t \geq 1 .
$$

From Theorem A we know that for some $a \in \mathbb{R}$,

$$
\frac{x(t)}{t} \rightarrow a \text { for } t \rightarrow \infty
$$


We have

$$
\int_{1}^{\infty} f(r, x(r)) d r<\infty
$$

since

$$
|f(r, x(r))| \leq h_{1}(r) \cdot g\left(\left|\frac{x(r)}{r}\right|\right)+h_{2}(r), \quad r \geq 1,
$$

and $\frac{x(r)}{r}$ is bounded on $[1, \infty)$.

We analyse the expression

$$
\begin{aligned}
\int_{1}^{t}\left[\int_{1}^{s} f(r, x(r)) d r\right] d s & \left.=\int_{1}^{t} \int_{1}^{\infty} f(r, x(r)) d r-\int_{s}^{\infty} f(r, x(r)) d r\right) d s \\
& =\int_{1}^{t} \int_{1}^{\infty} f(r, x(r)) d r d s-\int_{1}^{t} \int_{s}^{\infty} f(r, x(r)) d r d s \\
& =(t-1) \cdot \int_{1}^{\infty} f(r, x(r)) d r-\int_{1}^{t} \int_{s}^{\infty} f(r, x(r)) d r d s
\end{aligned}
$$

Letting $\chi_{[1, t]}$ be the characteristic function of the interval $[1, t]$, we have that for all $t \geq 1$,

$$
\begin{aligned}
\chi_{[1, t]}(s) \cdot\left|\int_{s}^{\infty} f(r, x(r)) d r\right| & \leq \int_{s}^{\infty}|f(r, x(r))| d r \\
& \leq \int_{s}^{\infty} h_{1}(r) \cdot g\left(\frac{|x(r)|}{r}\right) d r+\int_{s}^{\infty} h_{2}(r) d r \\
& \leq \sup _{r \geq 1} g\left(\frac{|x(r)|}{r}\right) \cdot \int_{s}^{\infty} h_{1}(r) d r+\int_{s}^{\infty} h_{2}(r) d r .
\end{aligned}
$$

By the Lebesgue dominated convergence theorem we obtain that the following limit exists and is finite

$$
\lim _{t \rightarrow \infty} \int_{1}^{t}\left(\int_{s}^{\infty} f(r, x(r)) d r\right) d s=l<\infty
$$

Hence, with

$$
K_{0}=x(1)-x^{\prime}(1)-\int_{1}^{\infty} f(r, x(r)) d r
$$


we get

$$
\begin{aligned}
& x(t)-\left[x^{\prime}(1)+\int_{1}^{\infty} f(r, x(r)) d r\right] \cdot t \\
& =x(1)-x^{\prime}(1)-\int_{1}^{\infty} f(r, x(r)) d r-\int_{1}^{t}\left(\int_{s}^{\infty} f(r, x(r)) d r\right) d s \\
& =K_{0}-\int_{1}^{t}\left(\int_{s}^{\infty} f(r, x(r)) d r\right) d s \stackrel{t \rightarrow \infty}{\longrightarrow} K_{0}-l,
\end{aligned}
$$

and therefore $x(t)=a t+b+o(1)$ as $t \rightarrow \infty$.

With respect to the sharpness of Theorem 2, the following examples are relevant.

EXAMPLE 4. Consider the equation

$$
u^{\prime \prime}+t^{\sigma} \operatorname{sgn}(u) \ln (1+|u|)=0, \quad t \geq 1
$$

where $\sigma \in \mathbb{R}$. For every $\epsilon \in(0,1)$, we have

$$
\ln (1+|x|) \leq \frac{1}{\epsilon}(1+|x|)^{\epsilon}, \quad x \in \mathbb{R},
$$

so that

$$
t^{\sigma} \ln (1+|u|) \leq \frac{1}{\epsilon} t^{\sigma+\epsilon}\left(1+\frac{|u|}{t}\right)^{\epsilon}, \quad t \geq 1, u \in \mathbb{R} .
$$

Since the function $g(r)=(1+r)^{\epsilon}, r \geq 0$ is nondecreasing with

$$
\int_{1}^{\infty} \frac{\mathrm{ds}}{g(s)}=\infty
$$

we can apply Theorem 2, if $\sigma+\epsilon<-2$. Due to the arbitraryness of $\epsilon \in(0,1)$, we conclude that if $\sigma<-2$, then for any $b \in \mathbb{R}$ there exists a solution to (9) with

$$
u(t)=b+o(1) \quad \text { as } \quad t \rightarrow \infty .
$$

Interestingly, the necessary and sufficient condition for (9) to fulfill (10) is $\sigma<-2$, cf. [9], despite the fact that all solutions of (9) are bounded if $\sigma=0$ (see [3], [11]).

EXAMPLE 5. Let us consider the sublinear Emden-Fowler equation

$$
u^{\prime \prime}=t^{\sigma} \cdot|u|^{\lambda-1} u, \quad t \geq 1,
$$

with $\lambda \in(0,1)$ and $\sigma<0$. If

$$
\sigma+\lambda+2<0
$$


then, as a consequence of Theorem 1 (with $g(r)=r^{\lambda}, r \geq 0$, and $h(s)=s^{\lambda+\sigma}, s \geq 1$ ) we have that for every real pair $(a, b)$, there is a solution of $(11)$ of the form

$$
u(t)=a t+b+o(1) \quad \text { as } \quad t \rightarrow \infty .
$$

Moreover, Theorem 2 guarantees that every solution of (11) is of the above form for some $a, b \in \mathbb{R}$, provided (12) holds.

It is of interest to note that under the same assumption (12), the recent results in [7] are less accurate: they show only that every positive solution of (11) has one of the asymptotic forms

$$
u(t)=a t+o(t) \quad \text { as } \quad t \rightarrow \infty
$$

or

$$
u(t)=b+o(1) \quad \text { as } \quad t \rightarrow \infty
$$

where $a, b$ are some positive constants.

\section{REFERENCES}

1. D. S. Cohen, The asymptotic behaviour of a class of nonlinear differential equations, Proc. Amer. Math. Soc. 18 (1967), 607-609.

2. A. Constantin, On the asymptotic behaviour of second order nonlinear differential equations, Rend. Mat. Appl. 13 (1993), 627-634.

3. A. Constantin, A note on a second-order nonlinear differential system, Glasgow Math. J. 42 (2000), 195-199. 1964).

4. R. Conti and G. Sansone, Non-linear differential equations (Pergamon Press, London,

5. F. M. Dannan, Integral inequalities of Gronwall-Bellman-Bihari type and asymptotic behaviour of certain second order nonlinear differential equations, J. Math. Anal. Appl. 108 (1985), 151-164.

6. Fan Wei Meng, A note on Tong paper: The asymptotic behaviour of a class of nonlinear differential equations of second order, Proc. Amer. Math. Soc. 108 (1990), 383-386.

7. K. Kamo and H. Usami, Asymptotic forms of positive solutions of second-order quasilinear ordinary differential equations with sub-homogeneity, Hiroshima Math. J. 31 (2001), 35-49.

8. O. Lipovan, A retarded Gronwall-like inequality and its applications, J. Math. Anal. Appl. 252 (2000), 389-401.

9. M. Naito, Asymptotic behaviour of solutions of second order differential equations with integrable coefficients, Trans. Amer. Math. Soc. 282 (1984), 577-588.

10. V. Nemyckii, The fixed point method in analysis, Amer. Math. Soc. Transl. 34 (1963), $1-37$.

11. C. Qian, Boundedness and asymptotic behaviour of solutions of a second-order nonlinear systen, Bull. London Math. Soc. 24 (1992), 281-288.

12. S. Rogovchenko and Y. Rogovchenko, Asymptotic behaviour of solutions of second order nonlinear differential equations, Portugal. Math. 57 (2000), 17-33.

13. J. Tong, The asymptotic behaviour of a class of nonlinear differential equations of second order, Proc. Amer. Math. Soc. 84 (1982), 235-236.

14. W. Trench, On the asymptotic behaviour of solutions of second order linear differential equations, Proc. Amer. Math. Soc. 16 (1963), 12-16. 